\title{
Tomographic Structural Changes of the Inner Retina after Internal Limiting Membrane Peeling for Idiopathic Epiretinal Membrane
}

\author{
Ga Hee Han ${ }^{1}$, Dong Jin Han ${ }^{1}$, Jong Hyun Lee ${ }^{1}$, Suk Ho Byeon ${ }^{2}$, Joo Youn Shin ${ }^{1,2}$ \\ ${ }^{I}$ Department of Ophthalmology, Ilsan Paik Hospital, Inje University College of Medicine, Goyang, Korea \\ ${ }^{2}$ Department of Ophthalmology, Institute of Vision Research, Yonsei University College of Medicine, Seoul, Korea
}

\begin{abstract}
Purpose: To investigate the tomographic structural changes in the retinal layers after internal limiting membrane (ILM) peeling for idiopathic epiretinal membrane (ERM).

Methods: Sixty-nine eyes treated with vitrectomy and ILM peeling for idiopathic ERM were analyzed. Parafoveal retinal thickness was measured at baseline and 6 months after surgery.

Results: Total retinal thickness decreased significantly in the nasal and temporal subfields after surgery $(p<$ 0.001), whereas the inner nuclear layer and outer nuclear layer showed nasal thickening (all, $p<0.001$ ). The postoperative temporal/nasal subfield thickness ratio of each layer was significantly lower than that of fellow eyes. Eyes with larger ILM peeling showed a significantly lower temporal/nasal subfield thickness ratio ( $p=$ $0.033)$ than those with smaller sizes.

Conclusions: The retinal thickness of each layer showed anatomical changes from ILM peeling and ERM removal. Nasal parafoveal thickening and temporal thinning occurred in the inner retinal architecture, which might be affected by ILM peeling size.
\end{abstract}

Key Words: Epiretinal membrane, Internal limiting membrane, Optical coherence tomography, Retinal layer segmentation, Retinal nerve fiber layer

Idiopathic epiretinal membrane (ERM) can cause visual impairment when the macular or perimacular region is involved; vitrectomy and ERM removal either with or without internal limiting membrane (ILM) peeling are standard treatment procedures for ERM [1]. ILM has been considered as a scaffold for cellular proliferation on which glial cells may migrate to further create a tangential contractile force [2]. ILM peeling has been suggested to be effective at

Received: October 22, 2019 Final revision: January 10, 2020

Accepted: March 11, 2020

Corresponding Author: Joo Youn Shin, MD. Department of Ophthalmology, Ilsan Paik Hospital, Inje University College of Medicine, 170 Juhwaro, Ilsanseo-gu, Goyang 10380, Korea. Tel: 82-31-910-7240, Fax: 82-31911-7241, E-mail: I0377@paik.ac.kr reducing the risk of ERM recurrence by eliminating areas where cells, including myofibroblasts, can proliferate $[1,3]$. ILM peeling is therefore widely accepted to be an effective treatment option for eyes with ERM.

However, there are concerns that ILM peeling may cause retinal changes, including postoperative swelling of the arcuate retinal nerve fiber layer (RNFL) [4], inner retinal dimples [5], and reduction of the temporal peripapillary RNFL thickness [6-9]. Thinning of the retina in the temporal subfield and thickening of the nasal subfield of the macula due to nasal displacement of the retina have also been reported [10-17]. These processes have been suggested as secondary changes resulting from axonal transport and contractility alterations in the RNFL due to apoptotic 
and atrophic degeneration in the peripapillary area [18]; however, the mechanism and related factors for these processes are not definitively known.

Recent improvements in optical coherence tomography (OCT) and automated image analysis have enabled independent measurement of each retinal layer thickness, both objectively and quantitatively [19-22]. In the present study, we investigated tomographical structural changes of each retinal layer in eyes undergoing ILM peeling for idiopathic ERM. To the best of our knowledge, no studies have assessed the changes in each individual retinal layer after ILM peeling for idiopathic ERM. In particular, we focused on thickening of the nasal subfield and thinning of the temporal subfield and analyzed the factors affecting these processes.

\section{Materials and Methods}

\section{Ethics statement}

This retrospective interventional case series study was performed at a single center from October 2012 to June 2014. The research adhered to the tenets of the Declaration of Helsinki, and approval for the study was granted by the institutional review board/ethics committee of Yonsei University, Gangnam Severance Hospital (3-2014-0283). All patients who participated provided written informed consent.

\section{Patients and surgeries}

We analyzed patients who underwent successful ILM peeling using indocyanine green dye for idiopathic ERM and who had completed a minimum follow-up period of 6 months. The surgeries were performed by a single surgeon (SHB) using the same 25-gauge, 3-port pars plana technique (Constellation; Alcon Surgical, Fort Worth, TX, USA). We excluded 1) eyes with myopia $>6$ diopters or evidence of pathological myopia, 2) patients with glaucoma or optic nerve disorder, 3) eyes with any macular pathology other than ERM that could affect retinal thickness, 4) patients who had previously undergone vitrectomy, 5) eyes with postoperative complications, including elevated intraocular pressure $>21 \mathrm{mmHg}$ during the follow-up, 6) patients with significant media opacity, and 7) eyes with any macular or optic nerve disease in the opposite eye that might affect retinal thickness, including ERM or glaucoma. The ILM peeling size was measured using surgical video images, and the eyes were divided into two groups as follows: a smaller size (2-3 disc diameters [DDs] from the fovea) and a larger size (ILM peeling size $>3$ DDs).

\section{OCT measurements}

The main outcome of this study involved possible changes in each retinal layer's thickness 6 months after vitrectomy with ILM peeling for idiopathic ERM. Spectral-domain OCT (Spectralis HRA+OCT; Heidelberg Engineering, Franklin, MA, USA) was used to obtain macular volume scans preoperatively and again 6 months after surgery. The volume scans of more than 49 sections ( $<20 \mu \mathrm{m}$ spacing) of $20^{\circ} \times 20^{\circ}$ areas of the macula were examined. The quality of the scans was assessed before the analysis, and poor-quality images were excluded.

The retinal segmentation software that accompanied the Spectralis OCT instrument was used to identify each retinal layer and to quantitate its thickness. For the analysis, the retinal layers were divided into RNFL, ganglion cell layer (GCL), inner plexiform layer (IPL), inner nuclear layer (INL), outer plexiform layer (OPL), outer nuclear layer $(\mathrm{ONL})$, and photoreceptor layer (PhR; from the external limiting membrane to Bruch's membrane). All segmentation images were reviewed, and significant segmentation errors were manually modified. The software in the Spectralis instrument automatically calculated the average retinal thickness of each retinal layer in every standard retinal subfield of the central, superior, temporal, inferior, and nasal quadrants of the inner and outer rings as defined in the Early Treatment Diabetic Retinopathy Study [23]. The diameter of the central ring was $1 \mathrm{~mm}$; the inner ring's diameter was $3 \mathrm{~mm}$, and that of the outer ring was $6 \mathrm{~mm}$ (Fig. 1A, 1B). Our analysis focused on the retinal thickness of the inner ring.

\section{Statistical methods}

Patient characteristics, including age, sex, spherical equivalent (SE), and best-corrected visual acuity, were retrieved from the patients' medical charts. Changes from baseline to 6 months postoperatively in each retinal layer were compared using the paired $t$-test. The temporal/nasal 


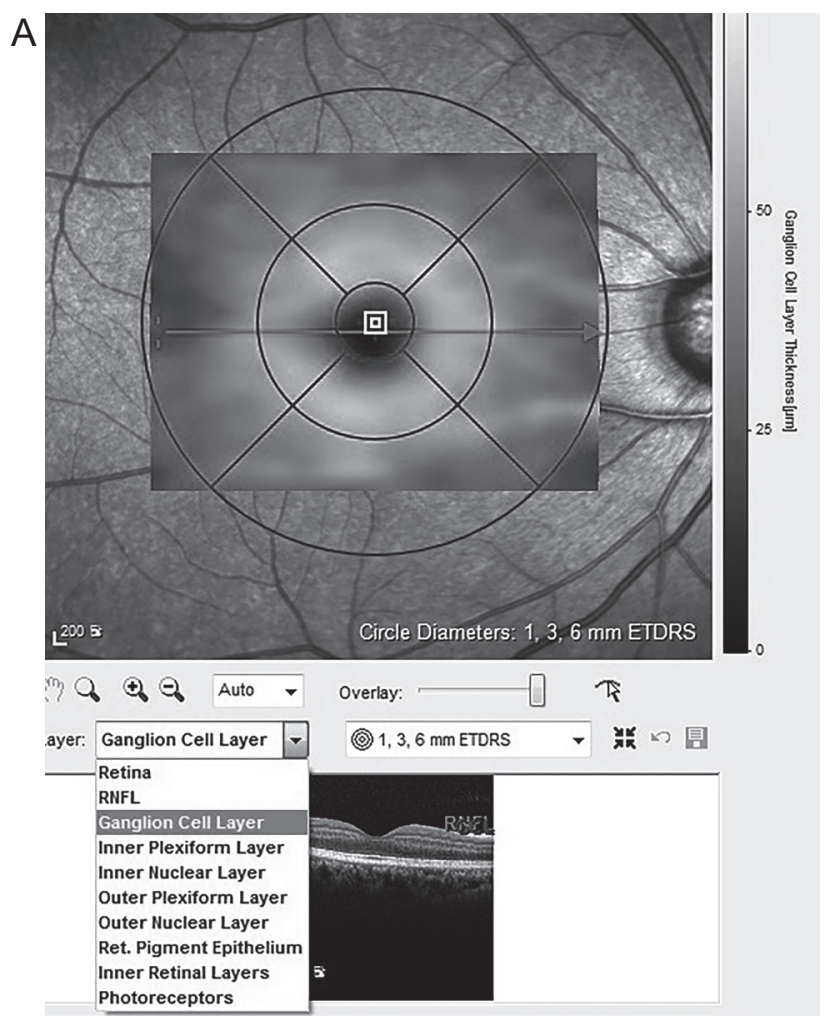

$B$

Average thickness $[\mu \mathrm{m}]$

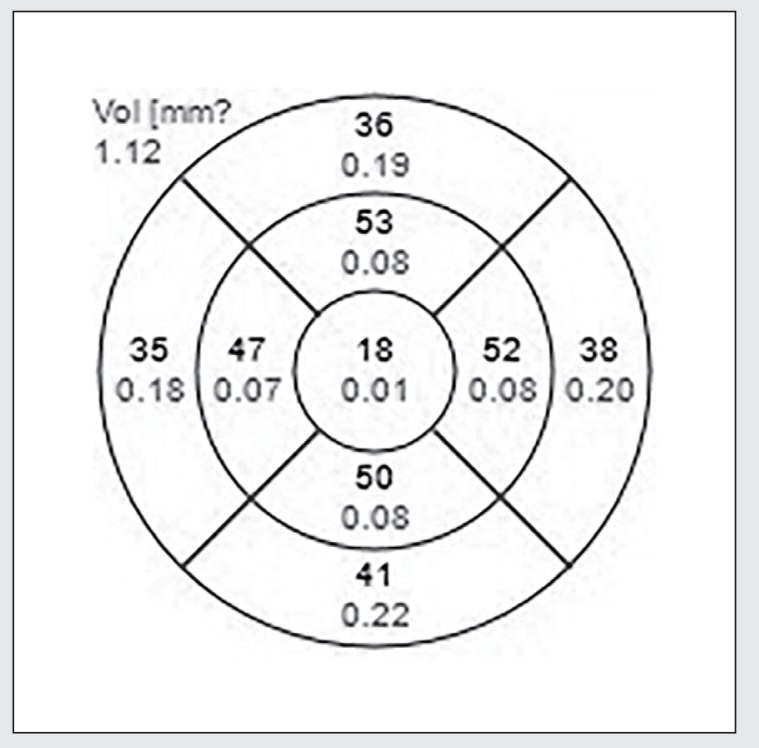

Fig. 1. Automated measurement of each retinal layer thickness using the segmentation application accompanying the Spectralis optical coherence tomography. (A) The software automatically marked each retinal layer and calculated its thickness. This figure shows ganglion cell layer thickness measurements using the segmentation software. (B) A macular thickness map using standard Early Treatment Diabetic Retinopathy Study (ETDRS) circles of 1,3 , and $6 \mathrm{~mm}$ showing the mean thickness of the ganglion cell layer in each of the nine subfields. In this study, the average thicknesses of the inner subfields were included in the analysis. ratio (TNR) was defined as the thickness in the temporal quadrant of the inner ring/nasal quadrants of the inner ring. Postoperative TNRs were compared with preoperative TNRs of affected eyes; for TNRs of the normal fellow eye, a paired $t$-test was used. Correlations between postoperative TNR of the entire retina and any other influencing variables were evaluated using Pearson's correlation. Statistical analyses were performed using the IBM SPSS Statistics ver. 20.0 (IBM Corp., Armonk, NY, USA), with a value of $p<0.05$ indicating statistical significance.

\section{Results}

Sixty-nine consecutive eyes in 69 patients were included in the analysis. The mean age was $65.9 \pm 8.3$ years, and 45 patients $(65.2 \%)$ were female. The mean preoperative SE was $0.08 \pm 1.56$ diopters. The participants' vision improved from $0.28 \pm 0.21$ to $0.11 \pm 0.11$ logarithm of the minimum angle of resolution after the surgery, and the mean retinal thickness within the inner ring ( $3 \mathrm{~mm}$ in diameter) decreased from $420.6 \pm 56.7$ to $362.9 \pm 27.8 \mu \mathrm{m}$. The size of the peeled ILM was $2-3$ DDs in 52 eyes (75.4\%) and $>3$ DDs in 17 eyes (24.6\%).

\section{Comparison between preoperative and postoperative retinal thicknesses}

Total retinal thickness significantly decreased both in the nasal and temporal inner subfields after surgery ( $p<$ $0.001)$. In terms of each retinal layer, the RNFL, IPL, and OPL showed thinning in both nasal ( $p<0.001,0.001$, and 0.002 , respectively) (Table 1) and temporal subfields (all, $p<0.001)$. The GCL thickness of the temporal subfield decreased significantly $(p<0.001)$, but the nasal subfield showed borderline significance $(p=0.05)$. However, the INL of the nasal subfield increased significantly from 49.7 \pm 7.5 to $55.1 \pm 8.9 \mu \mathrm{m}(p<0.001)$, with temporal thinning from $49.5 \pm 8.8$ to $43.3 \pm 7.4 \mu \mathrm{m}(p<0.001)$. The ONL also demonstrated nasal thickening from $75.5 \pm 17.6$ to $86.3 \pm$ $14.8 \mu \mathrm{m}(p<0.001)$, but the amount of temporal thinning was not significant $(p=0.81)$.

Comparison of the subfield TNRs in affected and fellow eyes 
The TNRs were compared using preoperative and postoperative measurements in affected eyes and also between postoperative measurements in the affected and fellow eyes. Compared with the preoperative TNR, the postoperative value was significantly lower in the total retina ( $p<$ $0.001)$, GCL $(p<0.001)$, IPL $(p<0.001)$, INL $(p<0.001)$, and ONL ( $p=0.002)$, while the RNFL ( $p=0.10)$, OPL ( $p$ $=0.30)$, and $\operatorname{PhR}(p=0.17)$ showed no significant difference. The postoperative TNR was significantly lower in the total retina $(p<0.001)$, RNFL $(p=0.005)$, GCL ( $p<$ $0.001)$, IPL $(p<0.001)$, INL $(p<0.001)$, and OPL $(p=$ $0.047)$ compared with those of normal fellow eyes. Howev- er, ONL $(p=0.09)$ and PhR showed no significant difference $(p=0.17)$ (Table 2).

\section{Factors associated with the TNR}

No correlation was found between TNR and age ( $p=$ $0.77)$, sex $(p=0.38)$, SE $(p=0.85)$, duration of symptoms $(p=0.61)$, preoperative retinal thickness $(p=0.970)$, or changes in the visual acuity $(p=0.63)$. Eyes with a larger ILM peeling size demonstrated a significantly lower TNR ( $p=0.033)$ than eyes with a smaller ILM peeling size (Table 3).

Table 1. Comparison between preoperative and postoperative retinal thicknesses of each layer

\begin{tabular}{|c|c|c|c|c|c|c|}
\hline & \multicolumn{3}{|c|}{ Nasal } & \multicolumn{3}{|c|}{ Temporal } \\
\hline & Preoperative & Postoperative & $p$-value & Preoperative & Postoperative & $p$-value \\
\hline Total & $422.2 \pm 53.3$ & $391.1 \pm 32.8$ & $<0.001^{*}$ & $414.4 \pm 63.7$ & $337.8 \pm 33.0$ & $<0.001^{*}$ \\
\hline RNFL & $67.3 \pm 43.5$ & $29.4 \pm 21.2$ & $<0.001^{*}$ & $56.6 \pm 48.3$ & $21.4 \pm 18.5$ & $<0.001^{*}$ \\
\hline GCL & $58.5 \pm 7.3$ & $56.1 \pm 9.1$ & $0.040^{*}$ & $57.3 \pm 11.6$ & $42.8 \pm 8.3$ & $<0.001^{*}$ \\
\hline IPL & $49.0 \pm 6.8$ & $45.4 \pm 6.5$ & $0.001^{*}$ & $50.2 \pm 8.6$ & $36.0 \pm 6.1$ & $<0.001^{*}$ \\
\hline INL & $49.7 \pm 7.5$ & $55.1 \pm 8.9$ & $<0.001^{*}$ & $49.5 \pm 8.8$ & $43.3 \pm 7.4$ & $<0.001^{*}$ \\
\hline OPL & $40.3 \pm 6.9$ & $36.6 \pm 8.0$ & $0.002^{*}$ & $38.5 \pm 5.8$ & $33.5 \pm 4.4$ & $<0.001^{*}$ \\
\hline ONL & $75.5 \pm 17.6$ & $86.3 \pm 14.8$ & $<0.001^{*}$ & $80.7 \pm 23.9$ & $80.0 \pm 13.3$ & 0.810 \\
\hline $\mathrm{PhR}$ & $82.4 \pm 3.2$ & $82.5 \pm 3.0$ & 0.760 & $81.9 \pm 2.9$ & $82.6 \pm 4.0$ & 0.080 \\
\hline
\end{tabular}

RNFL = retinal nerve fiber layer; GCL $=$ ganglion cell layer; IPL $=$ inner plexiform layer; INL $=$ inner nuclear layer; OPL $=$ outer plexiform layer; $\mathrm{ONL}=$ outer nuclear layer; $\mathrm{PhR}=$ photoreceptor layer. ${ }^{*} p<0.05$.

Table 2. Comparison of the temporal/nasal ratios in affected and fellow eyes

\begin{tabular}{lccccc}
\hline & Pre-TNR & Post-TNR & FE-TNR & $\begin{array}{c}p \text {-value } \\
\text { (pre-post) }\end{array}$ & $\begin{array}{c}p \text {-value } \\
\text { (post-FE) }\end{array}$ \\
\hline Retina & $98.3 \pm 10.3$ & $86.7 \pm 8.8$ & $96.0 \pm 4.6$ & $<0.001^{*}$ & $<0.001^{*}$ \\
RNFL & $84.7 \pm 48.1$ & $75.0 \pm 17.7$ & $85.1 \pm 19.5$ & 0.102 & $0.005^{*}$ \\
GCL & $98.2 \pm 16.9$ & $77.3 \pm 15.8$ & $90.0 \pm 9.7$ & $<0.001^{*}$ & $<0.001^{*}$ \\
IPL & $103.5 \pm 18.4$ & $80.6 \pm 16.2$ & $98.1 \pm 8.9$ & $<0.001^{*}$ & $<0.001^{*}$ \\
INL & $100.4 \pm 17.5$ & $79.4 \pm 13.4$ & $94.1 \pm 10.5$ & $<0.001^{*}$ & $<0.001^{*}$ \\
OPL & $98.8 \pm 24.6$ & $95.2 \pm 20.6$ & $103.4 \pm 25.9$ & 0.297 & $0.047^{*}$ \\
ONL & $108.4 \pm 23.5$ & $95.6 \pm 24.6$ & $101.4 \pm 15.0$ & $0.002^{*}$ & 0.090 \\
PhR & $99.4 \pm 2.3$ & $100.2 \pm 4.2$ & $99.4 \pm 2.3$ & 0.174 & 0.170 \\
\hline
\end{tabular}

$\mathrm{TNR}=$ temporal/nasal ratio; pre-TNR = preoperative TNR; post-TNR = postoperative TNR; FE-TNR = TNR of the fellow eye; pre-post $=$ preoperative and postoperative in affected eyes; post-FE = postoperative in the affected and fellow eyes; RNFL = retinal nerve fiber layer; GCL = ganglion cell layer; IPL $=$ inner plexiform layer; INL $=$ inner nuclear layer; $\mathrm{OPL}=$ outer plexiform layer; $\mathrm{ONL}=$ outer nuclear layer; $\mathrm{PhR}=$ photoreceptor layer. ${ }^{*} p<0.05$. 
Table 3. Correlations between postop temporal/nasal ratios and surgical variables

\begin{tabular}{lcc}
\hline & $\begin{array}{c}\text { Correlation } \\
\text { coefficient }\end{array}$ & $p$-value \\
\hline Age & 0.036 & 0.769 \\
Sex & 0.108 & 0.376 \\
Spherical equivalent & 0.023 & 0.851 \\
Duration of symptoms & 0.063 & 0.606 \\
Preoperative VA & 0.014 & 0.911 \\
Postoperative VA & 0.034 & 0.779 \\
Changes in VA & -0.060 & 0.627 \\
Preoperative retinal thickness & -0.005 & 0.970 \\
Postoperative retinal thickness & 0.040 & 0.744 \\
ILM peeling size & -0.258 & $0.033^{*}$ \\
\hline
\end{tabular}

$\mathrm{VA}=$ visual acuity; ILM $=$ internal limited membrane. ${ }^{*} p<0.05$.

\section{Discussion}

In the present study, we used the automated segmentation software of the Spectralis OCT instrument to investigate changes in thickness of each retinal layer in eyes that underwent ILM peeling for idiopathic ERM. Nasal parafoveal thickening and temporal thinning of the inner retina were observed, reflecting various anatomical changes resulting from ILM peeling as well as changes due to ERM removal. We also showed that nasal parafoveal thickening and temporal thinning of the retina may be affected by large ILM peeling size, whereas no correlation was found between severity of ERM or visual improvement.

Analysis of each retinal layer thickness using automated segmentation revealed different changes in each layer. Reductions in RNFL, GCL, IPL, and OPL were observed in both the nasal and temporal subfields, while INL and ONL showed thickening of the nasal subfield and thinning of the temporal subfield. These results might have been caused by release of traction from the ERM after removal and induction of nasal displacement to the inner retina from ILM peeling [10-17].

Intraretinal changes can occur in the ERM due to retinal traction and underlying anatomical disturbances. The inner retina, especially the RNFL, GCL and IPL, were affected most highly by an ERM in a previous study [24]. In our results, reductions of RNFL, GCL, IPL, and OPL thicknesses were observed in both the nasal and temporal subfields after surgery, which could be attributed to resolution of traction after ERM removal. In addition, ILM peeling could induce thinning of the inner retina, as seen in patients with macular holes $[25,26]$. The ILM is the basement membrane of Müller cells, so ILM removal has been suggested to cause glial apoptosis due to removal of Müller cell plates and may be responsible for inner retinal thinning [18].

The INL and ONL showed nasal retinal thickening and temporal thinning after surgery, which suggests that the effect of nasal displacement in these layers might be greater than that of retinal thinning after ERM removal. Postoperative nasal parafoveal thickening and temporal thinning of the inner retina have been reported in eyes undergoing ILM peeling for ERM [10-14] and macular holes [15-17]. The mechanisms responsible for these processes have not been fully identified, but foveal displacement toward the optic disc and shortening of the papillofoveal distance might be responsible [15]. Because ILM contributes to the biochemical strength of the retina without contractile properties, these changes might be caused by retinal components, such as the RNFL, which is mainly comprised of microtubules and actin filaments. Loss of structural support from ILM peeling and RNFL thinning or alteration in retinal contractility may cause nasal parafoveal thickening and temporal parafoveal thinning after surgery.

The PhR did not undergo thinning or nasal subfield thickening after surgery, but changes in the inner retina were still apparent. During normal retinal development, the inner retina can slip along the outer plexiform layer with regard to the outer retina, while ONL cells have greater cohesiveness due to the tangential-lateral stiffness imparted by zona adherens junctions between the PhR and Müller cells, which form the the external limiting membrane [27]. Our results showed no significant difference in the TNRs in the ONL and PhR compared with their normal fellow eyes. In addition, in cases where the boundary between Henle's fiber and the ONL was observed using OCT (increased contrast between the edges of the PhR nuclei and the HFL axons) [28], thickening of the nasal ONL was mainly observed in Henle's fiber layer, while the actual outer nuclear layer was less affected.

We analyzed the factors that influence TNR changes and found that eyes with a larger ILM peeling size showed a 
lower TNR compared with eyes with a smaller ILM peeling size. This result indicated that the larger was the ILM peeling size, the greater was the tomographic change of the inner retina. A previous report of patients with macular holes showed that the size of the removed ILM area was strongly associated with the number of postoperative changes, including shortening of the disc-foveal distance and extent of the dissociated optic nerve fiber layer [29], which we also consistently found in eyes with idiopathic ERM. However, there was no correlation between TNR and other factors, including preoperative retinal thickness or visual outcome. The clinical significance of TNR changes or nasal displacement of the inner retina is not yet established. Additional studies investigating retinal function using electroretinography and studies with a long-term follow-up period and larger numbers of participants are needed to clarify the clinical significance of such changes.

There were several limitations in our study, including the small sample size, the short follow-up period, and the lack of a control group. There was also the possibility that some error in the outer retinal thickness measurements could have occurred because of inner foveal displacement regarding the outer foveal center. Preoperative foveal displacement (due to ERM) may also have interfered with measurements of the change resulting from ILM peeling. However, the ERM is one of the most common indications of ILM peeling, and the preoperative foveal microstructure is difficult to measure in eyes with macular hole; thus, it would also be helpful to evaluate the changes that result from ILM peeling. Further studies of these issues could have important implications for decisions regarding possible surgery in these patients.

In conclusion, retinal thickness measurements using automated segmentation software showed different changes in each retinal layer after ILM peeling for ERM, which might have resulted from various retinal alterations. Further studies including more patients with various pathologies will help to optimize customized treatments for ILM peeling and will aid in developing minimally traumatic techniques for ILM removal.

\section{Conflict of Interest}

No potential conflict of interest relevant to this article was reported.

\section{Acknowledgements}

This research was supported by the Korea Health Technology R \& D Project through the Korea Health Industry Development Institute funded by the Ministry of Health and Welfare, Republic of Korea (HI17C1567).

\section{References}

1. Park DW, Dugel PU, Garda J, et al. Macular pucker removal with and without internal limiting membrane peeling: pilot study. Ophthalmology 2003;110:62-4.

2. Snead DR, James S, Snead MP. Pathological changes in the vitreoretinal junction 1: epiretinal membrane formation. Eye (Lond) 2008;22:1310-7.

3. Kwok AK, Lai TY, Li WW, et al. Indocyanine green-assisted internal limiting membrane removal in epiretinal membrane surgery: a clinical and histologic study. Am J Ophthalmol 2004;138:194-9.

4. Clark A, Balducci N, Pichi F, et al. Swelling of the arcuate nerve fiber layer after internal limiting membrane peeling. Retina 2012;32:1608-13.

5. Spaide RF. "Dissociated optic nerve fiber layer appearance" after internal limiting membrane removal is inner retinal dimpling. Retina 2012;32:1719-26.

6. Yamashita T, Uemura A, Kita H, Sakamoto T. Analysis of the retinal nerve fiber layer after indocyanine green-assisted vitrectomy for idiopathic macular holes. Ophthalmology 2006;113:280-4.

7. Kim KY, Yu SY, Kim MS, et al. Changes of parafoveal retinal nerve fiber layer thickness analyzed by spectral-domain optical coherence tomography after pars plana vitrectomy. Retina 2013;33:776-84.

8. Balducci N, Morara M, Veronese C, et al. Retinal nerve fiber layer thickness modification after internal limiting membrane peeling. Retina 2014;34:655-63.

9. Lalezary M, Shah RJ, Reddy RK, et al. Prospective Retinal and Optic Nerve Vitrectomy Evaluation (PROVE) study: twelve-month findings. Ophthalmology 2014;121:1983-9.

10. Treumer F, Wacker N, Junge O, et al. Foveal structure and thickness of retinal layers long-term after surgical peeling of idiopathic epiretinal membrane. Invest Ophthalmol Vis Sci 2011;52:744-50.

11. Lee SB, Shin YI, Jo YJ, Kim JY. Longitudinal changes in retinal nerve fiber layer thickness after vitrectomy for 
epiretinal membrane. Invest Ophthalmol Vis Sci 2014;55:6607-11.

12. Kumagai K, Ogino N, Furukawa M, et al. Retinal thickness after vitrectomy and internal limiting membrane peeling for macular hole and epiretinal membrane. Clin Ophthalmol 2012;6:679-88.

13. Loiudice P, Pellegrini M, Montesel A, et al. Negative correlation between retinal displacement and ganglion cell layer thickness changes in eyes with epiretinal membrane. Eur J Ophthalmol 2019:1120672119894887.

14. Kumagai K, Hangai M, Ogino N. Progressive thinning of regional macular thickness after epiretinal membrane surgery. Invest Ophthalmol Vis Sci 2015;56:7236-42.

15. Ohta K, Sato A, Fukui E. Asymmetrical thickness of parafoveal retina around surgically closed macular hole. $\mathrm{Br} J$ Ophthalmol 2010;94:1545-6.

16. Ishida M, Ichikawa Y, Higashida R, et al. Retinal displacement toward optic disc after internal limiting membrane peeling for idiopathic macular hole. Am J Ophthalmol 2014;157:971-7.

17. Faria MY, Ferreira NP, Cristovao DM, et al. Tomographic structural changes of retinal layers after internal limiting membrane peeling for macular hole surgery. Ophthalmic Res 2018;59:24-29.

18. Pichi F, Lembo A, Morara M, et al. Early and late inner retinal changes after inner limiting membrane peeling. Int Ophthalmol 2014;34:437-46.

19. Huang Y, Danis RP, Pak JW, et al. Development of a semi-automatic segmentation method for retinal OCT images tested in patients with diabetic macular edema. PLoS One 2013;8:e82922.

20. Garcia-Martin E, Larrosa JM, Polo V, et al. Distribution of retinal layer atrophy in patients with Parkinson disease and association with disease severity and duration. Am J Ophthalmol 2014;157:470-8.

21. Martinez-de-la-Casa JM, Cifuentes-Canorea P, Berrozpe C, et al. Diagnostic ability of macular nerve fiber layer thickness using new segmentation software in glaucoma suspects. Invest Ophthalmol Vis Sci 2014;55:8343-8.

22. Garcia-Martin E, Polo V, Larrosa JM, et al. Retinal layer segmentation in patients with multiple sclerosis using spectral domain optical coherence tomography. Ophthalmology 2014;121:573-9.

23. Hee MR, Puliafito CA, Duker JS, et al. Topography of diabetic macular edema with optical coherence tomography. Ophthalmology 1998;105:360-70.

24. Hecht I, Yeshurun I, Bartov E, et al. Retinal layers thickness changes following epiretinal membrane surgery. Eye (Lond) 2018;32:555-62.

25. Nakamura T, Murata T, Hisatomi T, et al. Ultrastructure of the vitreoretinal interface following the removal of the internal limiting membrane using indocyanine green. Curr Eye Res 2003;27:395-9.

26. Wolf S, Schnurbusch U, Wiedemann P, et al. Peeling of the basal membrane in the human retina: ultrastructural effects. Ophthalmology 2004;111:238-43.

27. Springer AD, Hendrickson AE. Development of the primate area of high acuity, 3: temporal relationships between pit formation, retinal elongation and cone packing. Vis Neurosci 2005;22:171-85.

28. Lujan BJ, Roorda A, Knighton RW, Carroll J. Revealing Henle's fiber layer using spectral domain optical coherence tomography. Invest Ophthalmol Vis Sci 2011;52:1486-92.

29. Steel DH, Chen Y, Latimer J, et al. Does internal limiting membrane peeling size matter? $J$ Vitreoretin Dis 2017;1:2733. 